\title{
A double-blind randomized controlled trial of the local application of vancomycin versus ampicillin powder into the operative field for thoracic and/or lumbar fusions
}

\author{
Mikinobu Takeuchi, MD, PhD, ${ }^{1,2,5}$ Norimitsu Wakao, MD, PhD, ${ }^{1,3}$ Mitsuhiro Kamiya, MD, PhD, ${ }^{1,3}$ \\ Atsuhiko Hirasawa, MD, ${ }^{1,3}$ Kenta Murotani, PhD, ${ }^{4}$ and Masakazu Takayasu, MD, PhD ${ }^{1,2}$ \\ ${ }^{1}$ Spine Center; Departments of ${ }^{2}$ Neurological Surgery and ${ }^{3}$ Orthopedics Surgery; and ${ }^{4}$ Division of Biostatistics, Clinical Research \\ Center, Aichi Medical University Hospital, Nagakute; and 5Department of Spine Surgery, Aichi Spine Hospital, Inuyama, Aichi, \\ Japan
}

OBJECTIVE Retrospective studies have reported that the local application of vancomycin (VCM) powder into the operative field decreases the incidence of surgical site infection (SSI) in thoracic and/or lumbar fusion. Authors of the present study prospectively evaluated the effects of VCM in patients undergoing thoracic and/or lumbar fusion.

METHODS In this randomized double-blind trial, 230 patients undergoing thoracic and/or lumbar fusion were randomly assigned to the local administration of VCM (interventional group, $1 \mathrm{~g}$ ) or ampicillin (AMP; control group, $1 \mathrm{~g}$ ) into the surgical field. The primary outcome was SSI results within 1 year of surgery.

RESULTS The trial was prematurely stopped according to predetermined rules. The results showed one superficial infection $(0.9 \%$, Staphylococcus aureus) and one deep infection $(0.9 \%, S$. aureus) in the VCM group and two superficial infections (1.8\%, Staphylococcus epidermidis and culture negative) and one deep infection $(0.9 \%$, methicillin-resistant $S$. aureus) in the AMP group. No significant differences in infection rates were observed between the groups $(p=0.8)$.

CONCLUSIONS This double-blind randomized controlled trial demonstrated that the local application of VCM or AMP powder into the operative field in short thoracic and/or lumbar fusion procedures resulted in a similar incidence of SSI.

- CLASSIFICATION OF EVIDENCE Type of question: therapeutic; study design: randomized controlled trial; evidence: class III.

Clinical trial registration no.: UMIN000009377 (umin.ac.jp/ctr)

https://thejns.org/doi/abs/10.3171/2018.3.SPINE171111

KEYWORDS surgical site infection; methicillin-resistant Staphylococcus aureus; vancomycin; quality of life;

Staphylococcus epidermidis

I NFECTION rates after thoracic and/or lumbar fusion surgery are typically $2.8 \%$ to $10 \%$. The source of bacteria is primarily methicillin-resistant Staphylococcus aureus (MRSA). ${ }^{1}$ Patients infected with MRSA require bed rest and/or reoperation, including drainage. Previous retrospective studies have reported that the local application of vancomycin (VCM) powder into the operative field decreased severe surgical site infections (SSIs) in thoracic and/or lumbar fusions. ${ }^{11,12,14}$ In contrast, a prospective randomized controlled trial (RCT) has demonstrated that the application of VCM into the surgical wound was ineffective for surgical infections following spinal fusion surgery. ${ }^{16}$ Therefore, whether VCM absolutely reduces severe SSI in thoracic and/or lumbar fusion is uncertain. Local application of ampicillin (AMP) into the operative field in orthopedic surgery did not reduce SSIs in an RCT performed in 1989. ${ }^{13}$

We examined whether the local application of VCM versus AMP into the operative field reduced SSIs in a double-blind RCT.

ABBREVIATIONS AMP = ampicillin; JOABPEQ = Japanese Orthopaedic Association Back Pain Evaluation Questionnaire; MRSA = methicillin-resistant Staphylococcus aureus; $\mathrm{QOL}$ = quality of life; RCT = randomized controlled trial; SA = Staphylococcus aureus; SE = Staphylococcus epidermidis; SSI = surgical site infection; VCM = vancomycin.

SUBMITTED October 12, 2017. ACCEPTED March 15, 2018.

INCLUDE WHEN CITING Published online August 10, 2018; DOI: 10.3171/2018.3.SPINE171111. 


\section{- Classification of Evidence \\ Type of Question Therapeutic \\ Study Design Randomized controlled trial \\ Evidence Class III}

\section{Summary Statement}

In a heterogeneous group of patients undergoing lumbar and/ or thoracic fusion, this class III randomized controlled trial (RCT) failed to show a difference in surgical site infection (SSI) rates between patients who received vancomycin (VCM) powder and those in whom ampicillin (AMP) powder was sprinkled over the operative field.

\section{Classification of Evidence}

Precisely because they provide the strongest evidence upon which to base clinical decisions, RCTs are also the most difficult studies to conduct. Takeuchi and colleagues have accepted this challenge, and their resulting paper is immensely valuable to the neurosurgical community both in the areas in which it succeeds and in those in which it falls slightly short of perfection. Through no fault of the investigators, the two treatment arms in their study are not equally balanced with regard to potentially important prognostic features. This is the reason for conducting a randomized trial, but randomization doesn't always succeed, in the same way that flipping an "honest" coin 10 times doesn't always result in an equal number of heads and tails. Here, there are statistically significant differences in patient sex ( $53 \%$ vs $39 \%$ males in the VCM vs AMP groups, respectively, $p=0.004$ ) and operation time (203 vs 240 minutes, $p=0.04$ ) and a potentially clinically significant difference in the frequency of diabetes ( $23 \%$ vs $14 \%, p$ $=0.09$ ). While the impact of the sex difference is debatable, the longer operation time in the AMP group means that more patients in that treatment arm received a second dose of antibiotics. Coupled with the high frequency of diabetes in the VCM group, these imbalances may have given an unfair "advantage" to the AMP treatment arm unrelated to the value of the intervention. Because these imbalances were not accounted for in the analysis of the study (using multivariable analysis), the level of evidence for this RCT is reduced to class III. A second concern is the authors' interpretation of their results. They found 1-year infection rates of $1.8 \%$ vs $2.6 \%$ (VMC vs AMP). This corresponds to a relative risk of infection at 1 year of $0.66(95 \% \mathrm{Cl} 0.11-3.85)$. The authors assert that their trial "demonstrated that the local application of VCM or AMP powder ... resulted in a similar incidence of SSI." A more precise interpretation of their results is that they are consistent with as much as an $89 \%$ reduction in the infection rate (favoring VCM) or a 3.85 times greater infection rate (favoring AMP). Thus, despite all their best efforts, the authors' trial provides insufficient evidence upon which to base a conclusion. This failure is related to the imprecision of the study's estimate of effect size, or in other words, the large size of the confidence interval, which includes both a substantial benefit for VCM and a substantial detriment. The reason for this imprecision can be traced back to the authors' sample size calculation. Calculating the correct sample size is a simple mathematical procedure. Four variables (primarily) influence the calculation: the level of significance $(\alpha$, often set at 0.05$)$, the power (1- $\beta$, often set at $80 \%)$, the effect size, and the number of patients per treatment arm. If the first three variables are known, the sample size is easily obtained. Thus, the definition of a "positive" trial is contained within the sample size calculation. In this case, the authors set a very ambitious goal of reducing the infection rate from $5 \%$ (AMP group) to $0.2 \%$ (VCM group)-a $96 \%$ relative risk reduction. Based on previously published data, this seems overly optimistic. However, a more conservative but still ambitious goal, for example, reducing the infection rate from $5 \%$ to $2.5 \%$ (a $50 \%$ relative risk reduction), would have required 907 patients in each treatment arm. The definition of the target effect size depends not only on data from prior studies, but also on clinical judgment. The authors may reasonably have determined that only a large reduction in the infection rate (from $5 \%$ to $0.2 \%$ ) would be clinically compelling enough to drive a switch from AMP to VMC. The important statistical point is that the assumptions involved in sample size calculation are important. Often, they mean the difference between a "positive" and a "negative" study.

- Michael Glantz, MD

Hershey Medical Center Hershey, Pennsylvania

\section{Methods \\ Study Design}

This investigator-initiated trial was sponsored by the Aikeikai Foundation. No commercial support was received for the study. The institutional review board approved the study, which is registered with the University Medical Information Network (UMIN) Clinical Trial Registry (http://umin.ac.jp/ctr), and its registration no. is UMIN000009377. The patients provided written informed consent and could withdraw from the trial at any time.

This double-blind RCT assigned patients at an approximate 1:1 ratio to receive a sprinkling of either VCM (interventional group) or AMP powder (control group) into the operative field. Simple randomization was performed using a table of random numbers. Both patients and surgeons were blinded to the randomization until after the completion of a 1-year follow-up.

\section{Subjects}

The criteria for inclusion were subjects undergoing thoracic and/or lumbar fusion surgery. Surgical indications included degenerative spondylosis, spondylolisthesis, degenerative spondylolisthesis, idiopathic spine deformity, previous lumbar or thoracic and/or lumbar surgery, traumatic unstable spinal column fracture, or metastatic unstable spinal column fracture. The exclusion criteria were allergies to VCM or AMP.

\section{Protocol}

We created two groups, the VCM group (interventional group) and the AMP group (control group). All patients 


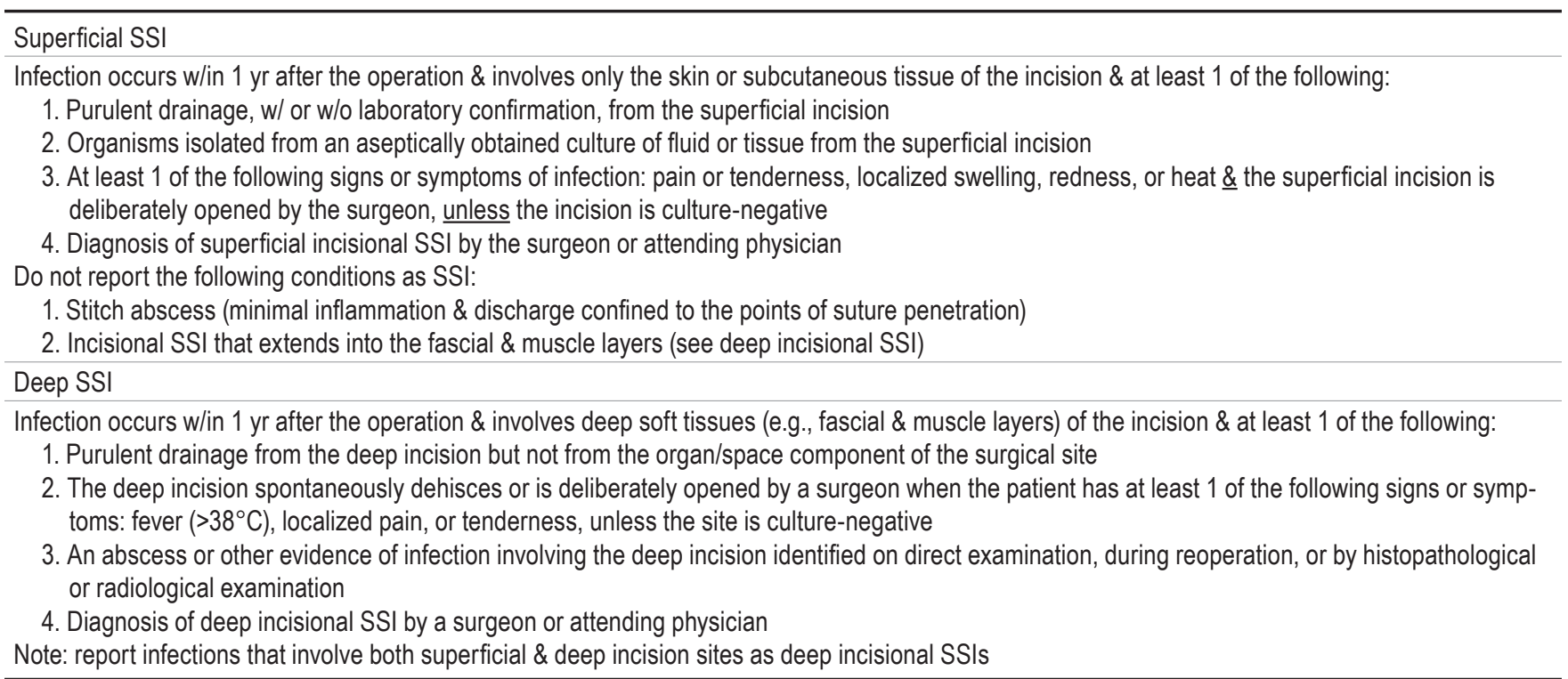

${ }^{*}$ As defined by the Centers for Disease Control and Prevention. Public domain.

received a first-generation $\beta$-lactam antibiotic intravenous drip $(1 \mathrm{~g})$ immediately before fusion surgery. The same antibiotic $(1 \mathrm{~g})$ was administered again if the surgery lasted more than 3 hours. Either VCM $(0.5 \mathrm{~g})$ or AMP $(0.5 \mathrm{~g})$ was mixed into the iliac or local bone grafts during the fusion procedure ${ }^{15}$ and VCM $(0.5 \mathrm{~g})$ or AMP $(0.5 \mathrm{~g})$ was distributed throughout the surgical wound after flushing the surgical field with $1 \mathrm{~L}$ of saline. For cases in which no bone grafts were used, VCM $(1 \mathrm{~g})$ or AMP $(1 \mathrm{~g})$ was applied to the surgical wound after flushing the surgical field.

The lumbar drainage tube was removed within fewer than 3 days after fusion surgery, and a first-generation $\beta$ lactam antibiotic intravenous drip (1 g) was initiated twice daily until removal of the lumbar drainage tube.

\section{Assessment of Clinical Outcomes}

The primary endpoint was the observation of deep or superficial SSI within 1 year of surgery, excluding remote infections such as postsurgical pneumonia, urinary tract infections, enteritis, or parotitis. If an SSI was suspected, blood sample collection, blood culture, and MRI were performed. Then, pus drainage was collected for bacterial culture. An SSI was defined according to the criteria established by the Centers for Disease Control and Prevention (Table 1). ${ }^{5,8}$

The secondary endpoints were 1-year postoperative scores on the Japanese Orthopaedic Association Back Pain Evaluation Questionnaire (JOABPEQ), ${ }^{2}$ which included quality of life (QOL) scores for three types of discomfort (low-back pain, leg pain, and leg numbness). The scores ranged from 0 to 100 , with higher scores indicating more pain. The patients answered 25 lumbago-specific questions. For additional QOL factors, the examiner calculated five functional scores (low-back pain, lumbar function, walking ability, social life function, and mental health) ranging from 0 to 100 , with higher scores indicating better QOL.

\section{Sample Size}

Power calculations were based on the results of a previous study. ${ }^{12}$ We hypothesized that the patients who received antibiotic application in the surgical field would exhibit a reduction in SSIs of $0.2 \%$ (VCM group) compared with a control effect of 5\% (AMP group). A sample size of 235 patients in each group was required to detect a minimum reduction of $0.2 \%$ in the VCM group for an alpha of 0.05 and a power of 0.80 , assuming a $10 \%$ dropout rate. According to predetermined rules, an intermediate analysis was performed when 120 patients reached the complete endpoint in each group. At that point, the steering committee decided to either continue or discontinue the study.

\section{Statistical Analysis}

The continuous variables are presented as the means with standard deviations. Results were analyzed using the Student t-test or the Kruskal-Wallis test for comparisons between the two groups and a paired t-test or the Wilcoxon signed-rank test for comparisons between pre- and postoperative scores. The categorical variables were analyzed using a chi-square test or Fisher's exact test. Statistical analyses were performed with SPSS software (version 22.0, IBM Corp.). The statistical significance level was set at less than 0.05 .

\section{Results}

This trial was discontinued at the intermediate stage because the intermediate results indicated that the final results would not yield a significant difference if the study continued until the sample size was achieved. A total of 265 patients at the hospital were enrolled from November 


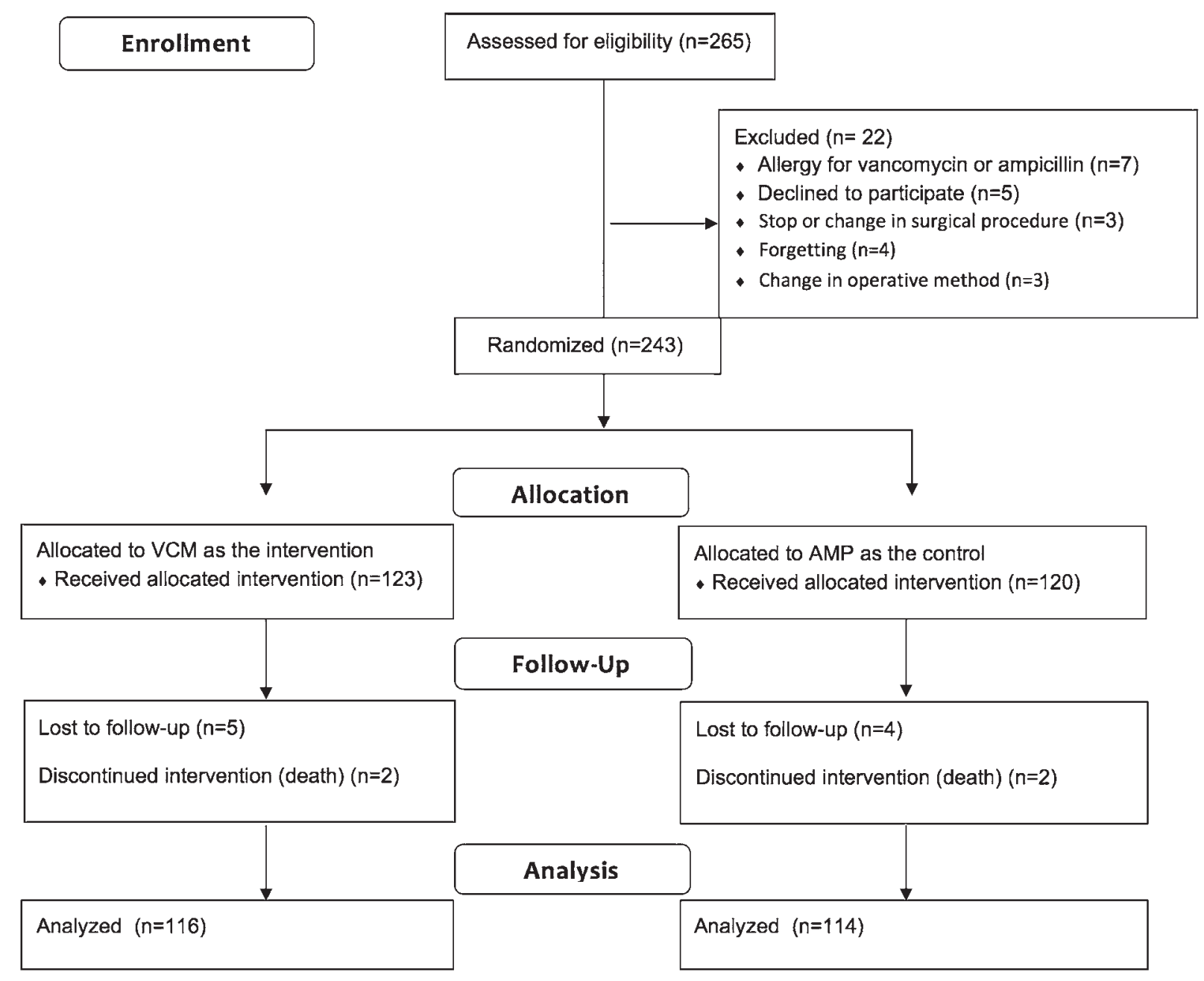

FIG. 1. Flow diagram of study patients.

2012 to December 2015, and 230 patients were randomly assigned to groups; 123 patients were allocated to receive $\mathrm{VCM}$ as the intervention group, and 120 patients received AMP as the control group. Five patients in the VCM group were lost to follow-up, and 2 patients died within 1 year after surgery. Four patients in the AMP group were lost to follow-up, and 2 patients died within 1 year after surgery. A total of 230 patients were ultimately included in our analysis (Fig. 1).

Table 2 presents the baseline demographics and clinical characteristics of both the VCM group (116 patients, including 61 men and 55 women, with a mean age of 66 years) and the AMP group (114 patients, including 44 men and 70 women, with a mean age of 68 years). The two groups did not significantly differ with respect to age, blood loss, operative time, number of fusion levels, diabetes status, smoking habits, BMI, previous thoracic and/ or lumbar surgery, steroid use, presence of trauma, or preoperative JOABPEQ scores, although they did differ in terms of patient sex $(\mathrm{p}=0.04)$.

One superficial infection $(0.9 \%$, Staphylococcus aureus [SA] ) and one deep infection $(0.9 \%, \mathrm{SA})$ were recorded in the VCM group, and two superficial infections (1.8\%,
Staphylococcus epidermidis [SE] and culture negative) and one deep infection (0.9\%, MRSA) were observed in the AMP group. No significant differences in the infection rates were observed between the groups $(\mathrm{p}=0.8$; Table 3). These infections were diagnosed at a median of 14 days (range 10-45 days) after surgery. No adverse postsurgical events were attributed to the application of VCM or AMP.

No differences in postoperative JOABPEQ scores were observed between the treatment groups (Table 4). All postoperative JOABPEQ scores were significantly improved compared to the preoperative scores in each of the groups $(\mathrm{p}=0.001 ;$ Table 5).

\section{Discussion}

In this double-blind RCT, we compared local VCM and local AMP application in the operative field in patients who underwent thoracic and/or lumbar fusion, finding no differences between the groups in terms of postoperative SSI rates 1 year after surgery. In addition, no differences in postoperative QOL assessment scores were observed between the groups. 
TABLE 2. Demographics and characteristics of patients who underwent thoracic and/or lumbar fusion

\begin{tabular}{lccl}
\hline \multicolumn{1}{c}{ Variable } & VCM Group & AMP Group & p Value \\
\hline No. of patients & 116 & 114 & \\
\hline Mean age in yrs & $66 \pm 14$ & $68 \pm 14$ & 0.1 \\
\hline Sex: M/W & $61 / 55$ & $44 / 70$ & $0.04^{*}$ \\
\hline Mean op time in mins & $203 \pm 101$ & $240 \pm 94$ & 0.3 \\
\hline Mean blood loss in ml & $429 \pm 629$ & $360 \pm 324$ & 0.95 \\
\hline Mean no. of fusion levels & $1.9 \pm 1.9$ & $2 \pm 1.7$ & 0.4 \\
\hline Diabetes & $27(23 \%)$ & $16(14 \%)$ & 0.09 \\
\hline Smoking & $32(28 \%)$ & $28(25 \%)$ & 0.7 \\
\hline Mean BMl in kg/m ${ }^{2}$ & $23.8 \pm 4$ & $23.3 \pm 4$ & 0.2 \\
\hline Previous thoracic \&/or lumbar & $11(9 \%)$ & $6(5 \%)$ & 0.1 \\
\hline surgery & & & \\
\hline Use of steroids & $4(3 \%)$ & $5(4 \%)$ & 0.8 \\
\hline Trauma & $11(9 \%)$ & $6(5 \%)$ & 0.1 \\
\hline Preop JOABPEQ (QOL score) & \multicolumn{3}{c}{} \\
\hline Degree of low-back pain & $61 \pm 27$ & $68 \pm 25$ & 0.2 \\
\hline Degree of leg pain & $66 \pm 25$ & $70 \pm 25$ & 0.5 \\
\hline Degree of leg numbness & $65 \pm 29$ & $64 \pm 30$ & 0.9 \\
\hline Low-back pain & $29(11,43)$ & $29(7,43)$ & 0.4 \\
\hline Lumbar function & $42(25,58)$ & $50(21,71)$ & 0.4 \\
\hline Walking ability & $21(7,50)$ & $29(7,43)$ & 0.9 \\
\hline Social life function & $36(22,52)$ & $38(15,51)$ & 0.8 \\
\hline Mental health & $39(27,51)$ & $39(26,50)$ & 0.7 \\
\hline
\end{tabular}

JOABPEQ = Japanese Orthopaedic Association Back Pain Evaluation Questionnaire.

Values are expressed as the mean \pm standard deviation, number (\%), or median (interquartile range).

*Indicates a significant difference between groups.

Retrospective studies on the local administration of VCM have reported that SSIs were reduced in patients with posterior spinal stabilization of traumatic injuries, ${ }^{12}$ instrumented thoracic and/or lumbar fusions, ${ }^{4,14}$ or spinal surgeries with or without fusion. ${ }^{11}$ However, one prospective RCT demonstrated that local VCM application did not significantly reduce SSIs for 3 months following thoracic and/or lumbar fusion surgery, ${ }^{16}$ and a second prospective study of posterior cervical fusion procedures demonstrated that the administration of VCM powder into the surgical field did not reduce SSIs within 30 days. ${ }^{10}$ While the results of these two studies were similar to our results, the present study provides higher-quality evidence than the previous prospective studies because ours was a doubleblind RCT and we examined SSI rates within 1 year after the thoracic and/or lumbar fusion surgery.

In our study, SSI rates were very low in both groups. In historical control data obtained prior to this RCT, we found an SSI rate of 4.1\% (5/123 patients) without any intraoperative local antibiotic administration. Infection rates were not significantly different in the historical control data, and rates of $1.7 \%$ in the VCM group and $2.6 \%$ in the AMP group were found in this study $(\mathrm{p}=0.8)$.

The postoperative JOABPEQ scores (degree of low-
TABLE 3. Infection rates and culture details

\begin{tabular}{lcc}
\hline \multirow{2}{*}{ Parameter } & \multicolumn{2}{c}{ No. of Patients (\%), Culture } \\
\cline { 2 - 3 } & VCM Group & AMP Group \\
\hline Total no. of patients & 116 & 114 \\
\hline Superficial infection & $1(0.9 \%)$, SA & $2(1.8 \%)$, SE \& CN \\
\hline Deep infection & $1(0.9 \%)$, SA & $1(0.9 \%)$, MRSA \\
\hline Total $^{*}$ & $2(1.7 \%)$ & $3(2.6 \%)$ \\
\hline CN = culture negative; SA = Staphylococcus aureus; SE = Staphylococcus \\
epidermidis. \\
${ }^{*} p=0.8$, VCM compared with AMP.
\end{tabular}

back pain, degree of leg pain, degree of leg numbness, low-back pain, lumbar function, walking ability, social life function, or mental health), as assessments of QOL, revealed no significant differences between the groups. All postoperative JOABPEQ scores were significantly better than the preoperative scores, and no adverse effects were reported in either group. Therefore, local application of VCM in the operative field did not affect the postoperative QOL assessment. No previous reports are available on the postoperative QOL assessments of patients who had local VCM application in the operative field, but some studies have reported adverse postoperative events. Ghobrial et al. conducted a systematic review examining adverse events in 9721 patients who had received intrawound VCM and reported 23 adverse events (0.3\%): 1 patient experienced nephropathy, 2 exhibited ototoxicity that resulted in transient hearing loss, 1 experienced systemic absorption that resulted in supratherapeutic VCM exposure, and 19 showed culture-negative seroma formation. ${ }^{3}$ These adverse events were very infrequent.

Several previous reports have indicated that the use of VCM powder in surgical wounds has expanded worldwide, which may increase the number of VCM-resistant bacteria., ${ }^{411,12,14}$ In fact, VCM-resistant enterococci were identified in the 1990s because of the increased unnecessary use of oral VCM. The increased and widespread use of intrawound VCM powder for spine fusion surgery may similarly increase the incidence of VCM-resistant bacteria. Therefore, this study contributes substantially to

TABLE 4. Postoperative JOABPEQ scores as the QOL assessment

\begin{tabular}{lccc}
\hline Postop JOABPEQ (QOL score) & VCM Group & AMP Group & p Value \\
\hline Degree of low-back pain & $22 \pm 23$ & $24 \pm 23$ & 0.8 \\
\hline Degree of leg pain & $24 \pm 25$ & $26 \pm 26$ & 0.7 \\
\hline Degree of leg numbness & $23 \pm 24$ & $29 \pm 32$ & 0.4 \\
\hline Low-back pain & $100(71,100)$ & $71(43,100)$ & 0.1 \\
\hline Lumbar function & $75(58,100)$ & $75(54,100)$ & 0.4 \\
\hline Walking ability & $86(57,100)$ & $64(43,100)$ & 0.2 \\
\hline Social life function & $73(51,86)$ & $68(51,89)$ & 0.4 \\
\hline Mental health & $63(51,73)$ & $60(45,72)$ & 0.3 \\
\hline
\end{tabular}

Values expressed as the mean \pm standard deviation or as the median (interquartile range). 
TABLE 5. Differences in preoperative and postoperative JOABPEC scores

\begin{tabular}{|c|c|c|c|c|}
\hline $\begin{array}{l}\text { JOABPEQ } \\
\text { (QOL score) }\end{array}$ & $\begin{array}{l}\text { VCM } \\
\text { Group }\end{array}$ & $\begin{array}{c}p \\
\text { Value }\end{array}$ & $\begin{array}{l}\text { AMP } \\
\text { Group }\end{array}$ & $\begin{array}{c}p \\
\text { Value }\end{array}$ \\
\hline Degree of low-back pain* & 38 (31 to 46$)$ & 0.001 & 44 (35 to 51$)$ & 0.001 \\
\hline Degree of leg pain* & 42 (34 to 49$)$ & 0.001 & 44 (36 to 51$)$ & 0.001 \\
\hline Degree of leg numbness* & $42(35$ to 49$)$ & 0.001 & 35 (27 to 44$)$ & 0.001 \\
\hline Low-back pain† & 50 (43 to 57$)$ & 0.001 & $43(36$ to 50$)$ & 0.001 \\
\hline Lumbar function† & 33 (25 to 38$)$ & 0.001 & 25 (17 to 30$)$ & 0.001 \\
\hline Walking ability $\dagger$ & 43 (32 to 50$)$ & 0.001 & 36 (25 to 43$)$ & 0.001 \\
\hline Social life function $†$ & 32 (26 to 39$)$ & 0.001 & 31 (24 to 38$)$ & 0.001 \\
\hline Mental health $\dagger$ & $22(17$ to 26$)$ & 0.001 & 22 (17 to 27$)$ & 0.001 \\
\hline
\end{tabular}

Boldface type indicates statistical significance.

* Values expressed as the difference between the mean preoperative and postoperative scores ( $95 \%$ confidence intervals).

$\dagger$ Values expressed as the difference between the median preoperative and postoperative scores ( $95 \%$ confidence intervals).

the literature because it shows that the local application of VCM powder in the surgical wound did not reduce SSIs in thoracic and/or lumbar fusion surgery.

\section{Study Limitations}

There are some limitations in the present study. First, we used AMP as the control drug for two reasons: the institutional review board did not accept the use of a placebo drug. In orthopedic surgery, the efficacy of primary SSI prophylaxis with local antibiotics is uncertain, ${ }^{6}$ and the local application of AMP in the operative field did not reduce SSIs in an RCT conducted in $1989 .{ }^{13}$ Therefore, we considered AMP as the control drug.

Second, this double-blind RCT hypothesized the superiority of VCM over AMP. This study may be more suitable for noninferiority trials than for superiority trials because these two drugs are active drugs. We did not conduct a noninferiority trial because the use of VCM in the operative field may have significantly reduced SSIs more than AMP prior to the start of this study.

Third, long fusion procedures, such as those for adult spinal deformity or adolescent idiopathic scoliosis, were infrequently performed in our hospital during the study period. Different infection rates may have been found between the groups if the number of long fusion cases had been higher. However, a recent retrospective study reported that the local application of VCM powder was not associated with a significant difference in the rate of deep SSIs after spinal deformity surgery. ${ }^{9}$

Fourth, this study was discontinued at the intermediate stage. The primary reason for early termination was the lack of government approval for the local application of VCM or AMP in the surgical field (intravenous drips were naturally approved). Therefore, if complications related to the application of VCM or AMP had occurred, ethical issues may have emerged. Fortunately, at the intermediate stage, no adverse postsurgical events were attributed to local VCM or AMP application. A statistician analyzed the intermediate results and indicated that the final results would not yield a significant difference if the study continued until the sample size was achieved. Therefore, the steering committee decided to stop the RCT.

Fifth, the sample size was small. Significant differences in SSI rates may have been found between the groups if the trial had been continued until 1000 patients were included in each group. However, we hypothesize that the clinical importance would have been very low.

Sixth, first-generation $\beta$-lactam antibiotic intravenous drips $(1 \mathrm{~g})$ were administered until removal of the lumbar drainage. This practice may not be popular in many hospitals.

\section{Conclusions}

This double-blind RCT suggests that the local application of either VCM or AMP powder in the operative field in short thoracic and/or lumbar fusion procedures results in similar rates of SSI.

\section{Acknowledgments}

We thank the following orthopedic doctor and pharmacists for their assistance with the study: Dr. Koji Yamada from Kanto Rosai Hospital and Mrs. Akiko Tsukada, Miss Nakamura Shinko, and Miss Akiko Fujii from Aichi Medical University.

This study was funded by the Aikeikai Foundation.

\section{References}

1. Abbey DM, Turner DM, Warson JS, Wirt TC, Scalley RD: Treatment of postoperative wound infections following spinal fusion with instrumentation. J Spinal Disord 8:278-283, 1995

2. Fukui M, Chiba K, Kawakami M, Kikuchi S, Konno S, Miyamoto M, et al: JOA Back Pain Evaluation Questionnaire (JOABPEQ)/JOA Cervical Myelopathy Evaluation Questionnaire (JOACMEQ). The report on the development of revised versions. April 16, 2007. The Subcommittee of the Clinical Outcome Committee of the Japanese Orthopaedic Association on Low Back Pain and Cervical Myelopathy Evaluation. J Orthop Sci 14:348-365, 2009

3. Ghobrial GM, Cadotte DW, Williams K Jr, Fehlings MG, Harrop JS: Complications from the use of intrawound vancomycin in lumbar spinal surgery: a systematic review. Neurosurg Focus 39(4):E11, 2015

4. Hill BW, Emohare O, Song B, Davis R, Kang MM: The use of vancomycin powder reduces surgical reoperation in posterior instrumented and noninstrumented spinal surgery. Acta Neurochir (Wien) 156:749-754, 2014

5. Horan TC, Gaynes RP, Martone WJ, Jarvis WR, Emori TG: CDC definitions of nosocomial surgical site infections, 1992: a modification of CDC definitions of surgical wound infections. Am J Infect Control 20:271-274, 1992

6. Huiras P, Logan JK, Papadopoulos S, Whitney D: Local antimicrobial administration for prophylaxis of surgical site infections. Pharmacotherapy 32:1006-1019, 2012

7. Luber AD, Jacobs RA, Jordan M, Guglielmo BJ: Relative importance of oral versus intravenous vancomycin exposure in the development of vancomycin-resistant enterococci. J Infect Dis 173:1292-1294, 1996

8. Mangram AJ, Horan TC, Pearson ML, Silver LC, Jarvis WR: Guideline for prevention of surgical site infection, 1999. Centers for Disease Control and Prevention (CDC) Hospital Infection Control Practices Advisory Committee. Am J Infect Control 27:96-134, 1999

9. Martin JR, Adogwa O, Brown CR, Bagley CA, Richardson 
WJ, Lad SP, et al: Experience with intrawound vancomycin powder for spinal deformity surgery. Spine (Phila Pa 1976) 39:177-184, 2014

10. Martin JR, Adogwa O, Brown CR, Kuchibhatla M, Bagley $\mathrm{CA}$, Lad SP, et al: Experience with intrawound vancomycin powder for posterior cervical fusion surgery. J Neurosurg Spine 22:26-33, 2015

11. Molinari RW, Khera OA, Molinari WJ III: Prophylactic intraoperative powdered vancomycin and postoperative deep spinal wound infection: 1,512 consecutive surgical cases over a 6-year period. Eur Spine J 21 (Suppl 4):S476-S482, 2012

12. O'Neill KR, Smith JG, Abtahi AM, Archer KR, Spengler $\mathrm{DM}, \mathrm{McGirt} \mathrm{MJ}$, et al: Reduced surgical site infections in patients undergoing posterior spinal stabilization of traumatic injuries using vancomycin powder. Spine J 11:641-646, 2011

13. Raahave D, Hesselfeldt P, Pedersen T, Zachariassen A, Kann $\mathrm{D}$, Hansen $\mathrm{OH}$ : No effect of topical ampicillin prophylaxis in elective operations of the colon or rectum. Surg Gynecol Obstet 168:112-114, 1989

14. Sweet FA, Roh M, Sliva C: Intrawound application of vancomycin for prophylaxis in instrumented thoracolumbar fusions: efficacy, drug levels, and patient outcomes. Spine (Phila Pa 1976) 36:2084-2088, 2011

15. Takeuchi M, Kamiya M, Wakao N, Hirasawa A, Kawanami $\mathrm{K}$, Osuka K, et al: Large volume inside the cage leading incomplete interbody bone fusion and residual back pain after posterior lumbar interbody fusion. Neurosurg Rev 38:573578,2015

16. Tubaki VR, Rajasekaran S, Shetty AP: Effects of using intravenous antibiotic only versus local intrawound vancomycin antibiotic powder application in addition to intravenous an- tibiotics on postoperative infection in spine surgery in 907 patients. Spine (Phila Pa 1976) 38:2149-2155, 2013

\section{Disclosures}

The authors report no conflict of interest concerning the materials or methods used in this study or the findings specified in this paper.

\section{Author Contributions}

Conception and design: Wakao, Takeuchi. Acquisition of data: Wakao, Takeuchi, Kamiya, Hirasawa. Analysis and interpretation of data: Murotani. Drafting the article: Takeuchi. Critically revising the article: Takeuchi. Reviewed submitted version of manuscript: Takeuchi. Statistical analysis: Murotani. Administrative/ technical/material support: Wakao. Study supervision: Wakao, Kamiya, Takayasu.

\section{Supplemental Information}

\section{Previous Presentations}

This work was presented at the 2018 AANS Annual Scientific Meeting held on April 28-May 2, 2018, in New Orleans, LA.

\section{Correspondence}

Norimitsu Wakao: Aichi Medical University, Nagakute City, Aichi, Japan.nwakao2009@yahoo.co.jp. 